\title{
The Design of Micro Vacuum Heating Observation Equipment
}

\author{
Gao Changqing $^{1, a}$, Zhang Jianhua ${ }^{1, b}$, Ma Chao ${ }^{1, c}$, Wang Mingyu ${ }^{1, d}$, Li \\ Jianmei ${ }^{1, e}$ \\ ${ }^{1}$ School of Shandong University, Jinan 250061, China; \\ agaochangqing666@126.com, bjhzhang@sdu.edu.cn, 'symachao10@163.com, \\ dmingyuwangsdu@hotmail.com, elijianmei@sdu.edu.cn
}

Keywords: vacuum, heater, observation.

\begin{abstract}
In order to study the transition of material properties under the environment of high temperature and vacuum, a micro vacuum heating observation equipment was designed. The vacuum system can create the vacuum environment. And the heating system can create the high temperature environment. This equipment was built by a combination of vacuum chamber and heater, which could decrease the space of device effectively and reach the focal length range of long distance microscope. Finally, the observation on the process of material organization and properties change under the high temperature and vacuum chamber could be achieved. The experiment was also conducted. It is found that this device could realize the observation under the environment of vacuum and heating process.
\end{abstract}

\section{Introduction}

With the development of the industry and the progress of science and technology, more and more materials were applied to the high temperature and vacuum environment, which speed up the development of vacuum heating equipment[1-3]. Zhang simulates the vacuum environment[1]. And Lian simulates the high temperature environment[2]. Gao designed an equipment to simulate materials in a composite environment[3]. However, these equipment mostly simulate in single environment. Equipment for the simulation of composite environment also be designed largely, because the heating equipment need to be placed in the vacuum chamber. These equipments can only observe the results after the change, the observation of changing process could not be monitored.

This paper introduced a vacuum heating observation equipment which could heat the sample under a vacuum environment and realize thereal-time observation of crack growth under different temperature conditions. The work-piece was placed in a vacuum chamber. The heating wire was winded on the outer wall of vacuum chamber and put them together. Thus the work-piece could be heated in a vacuum environment and the volume of equipment could be reduced effectively. There is an observation window on the right above of vacuum chamber. The lens of a microscope was installed here which could realize the observation. The device has the advantages of small size and flexible movement, and can be adapted to the focal length of the microscope.

\section{Construction}

\subsection{Overall Design}

The micro vacuum heating observation equipment is designed to simulate high temperature and vacuum environment. It is compact and user friendly. The degree of vacuum can reach $1 \times$ $10-3 \mathrm{~Pa}$.And the equipment can achieve a high temperature which is $700{ }^{\circ} \mathrm{C}$. The temperature is adjustable and the accuracy of measurement is within $5^{\circ} \mathrm{C}$. The time is required within thirty minutes for a certain vacuum and temperature degree. In order to reach the range of microscope, the distance between the bottom and window must be less than $70 \mathrm{~mm}$.

The main parts of the equipment include vacuum system, heating system and so on.Fig.1 is a diagram of the whole design. 


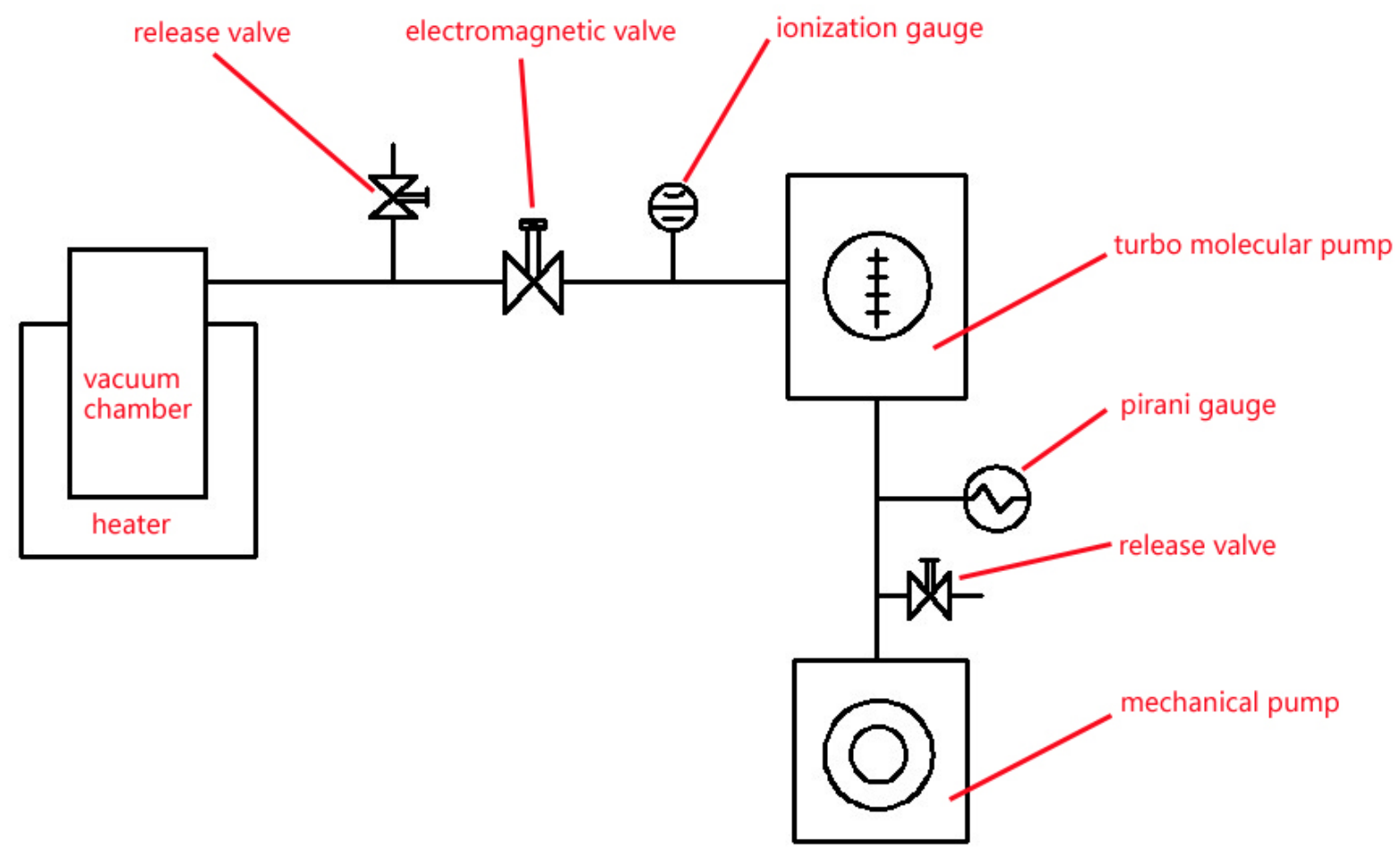

Fig. 1 Block diagram of the whole design

\subsection{Vacuum Chamber}

The vacuum chamber is one of the most important parts of the vacuum heating observation equipment. It provides the space where to place the work-piece. The shape of the vacuum chamber is designed to be cylindrical. In order to develop the relationship between the vacuum chamber and the observation window, six threaded holes was designed on the vacuum chamber. And the size is based on the CF flange size.

This equipment is mainly used to observe small work-pieces. This design is directed against a solid block of steel which the length of all sides is $15 \mathrm{~mm}$. Therefore the inner dimensions the vacuum need to be larger than $21 \mathrm{~mm}$. In order to make the operation more convenient, the inner diameter is designed $30 \mathrm{~mm}$. Connecting flange can choose CF35. Vacuum chamber materials can choose stainless steel $316 \mathrm{~L}$. The theoretical thickness of the vacuum chamber can be described by the following formula.

$$
S_{0}=1.25 D_{B}\left(\frac{p}{E_{t}} \cdot \frac{L}{D_{B}}\right)^{0.4}
$$

Where $S_{0}$-theoretical thickness of vacuum chamber

$D_{B}$-inner diameter of vacuum chamber

$p$-external pressure

$L$-the length of vacuum chamber

$E_{t}$-Modulus of Elasticity

The actually thickness of vacuum chamber is thicker than the theoretical thickness because of the additional thickness. The actually thickness can be described by the simple formula.

$S=S_{0}+C$

Where $S$-the actually thickness of vacuum chamber

$S_{0}$-theoretical thickness of vacuum chamber

$C$-additional thickness of vacuum chamber 
The additional thickness of vacuum chamber means the minus tolerance and atmospheric corrosion were taken into account. And it can be described by the formula.

$C=C_{1}+C_{2}$

Where $C$-additional thickness of vacuum chamber

$C_{1}$-the maximum of the minus tolerance

$C_{2}$-the thickness of atmospheric corrosion

Considering the vacuum chamber is used for precision measurement, the safety coefficient is chosen 2.5.Then the final thickness of vacuum chamber is $4 \mathrm{~mm}$. Fig.2 is the design of vacuum chamber.

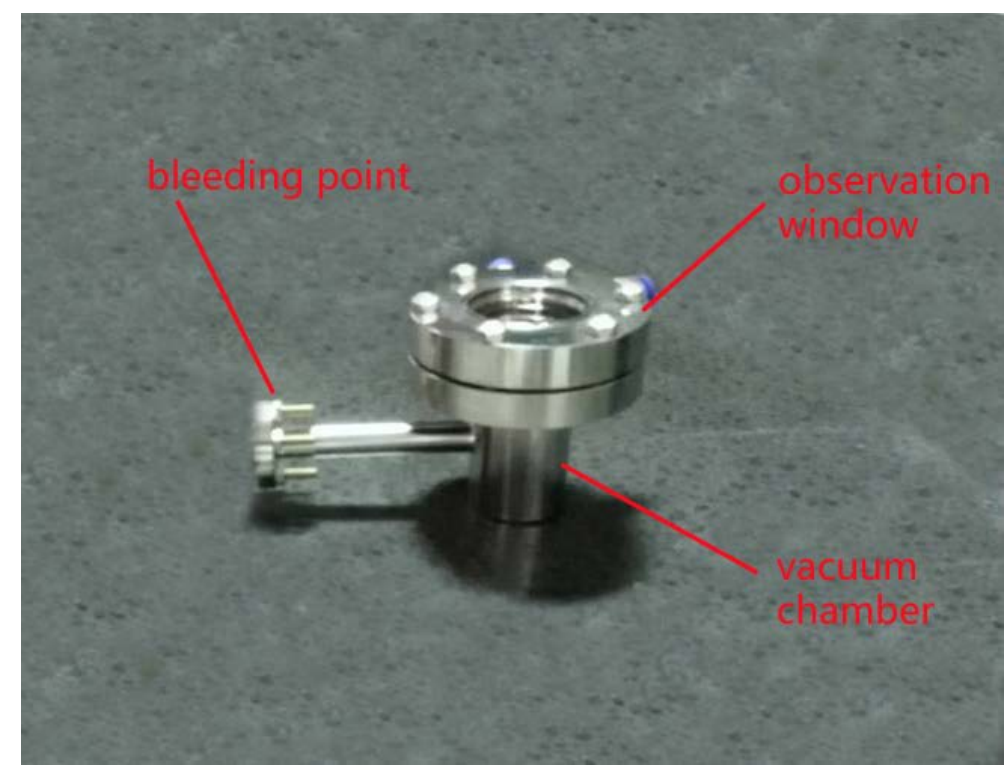

\subsection{Vacuum Connecting Line}

Fig. 2 The design of vacuum chamber

During the process of work, air enters the vacuum system constantly. In order to ensure the vacuum degree which is required, the vacuum pump should keep working. Because of the heating system, air will be with a high temperature. Overheating air has a negative effect on the vacuum pump. So a copper cooling pipe which will be put into cool water to low the air temperature is needed.

Metal corrugated pipe is very common in vacuum connecting line. Its sealing and shock resistance are good. It can reduce the vibration which is produced by the pump. So it has a good influence of the vacuum system. Because of the vacuum degree is not very high, we choose the metal corrugated to connect the vacuum pump and vacuum chamber.

At the exit of the vacuum chamber, a high vacuum electromagnetic valve is needed. The GDC-J40 is appropriate. At the end of the work, we should let the air get into the vacuum system. So we choose QF-5 manual vacuum release valve.

During the work, we should measure the vacuum degree at two time points. The first time point is when to open molecular pump and the second time point is when to close molecular pump. Therefore, ionization gauge, display and control circuit are needed.

\subsection{Vacuum Pump}

According to the vacuum degree, turbo molecular pump is suitable. It is cleaner than oil diffusion pump and it has more ability of heat-resistant. Turbo molecular pump's blades will be in a high-speed when it is working. As a result of the high-speed rotating blades, the air in the vacuum chamber is pumped. And then the system reaches the vacuum degree. However, turbo molecular pump can't start directly. It needs a backing pump. We choose the rotary vane mechanical pump.

\subsection{Heater}

In order to decrease the space of device effectively and reach the focal length range of long distance microscope, the device was built by a combination of vacuum chamber and heater. The 
heating wire was winded on the outer wall of vacuum chamber and put them together. Ceramic fiber is used for heat preservation and the metal shield can reduce the heat lose. At the bottom of the heater there is a water cooled pipe. Fig. 3 is the design of the heater.

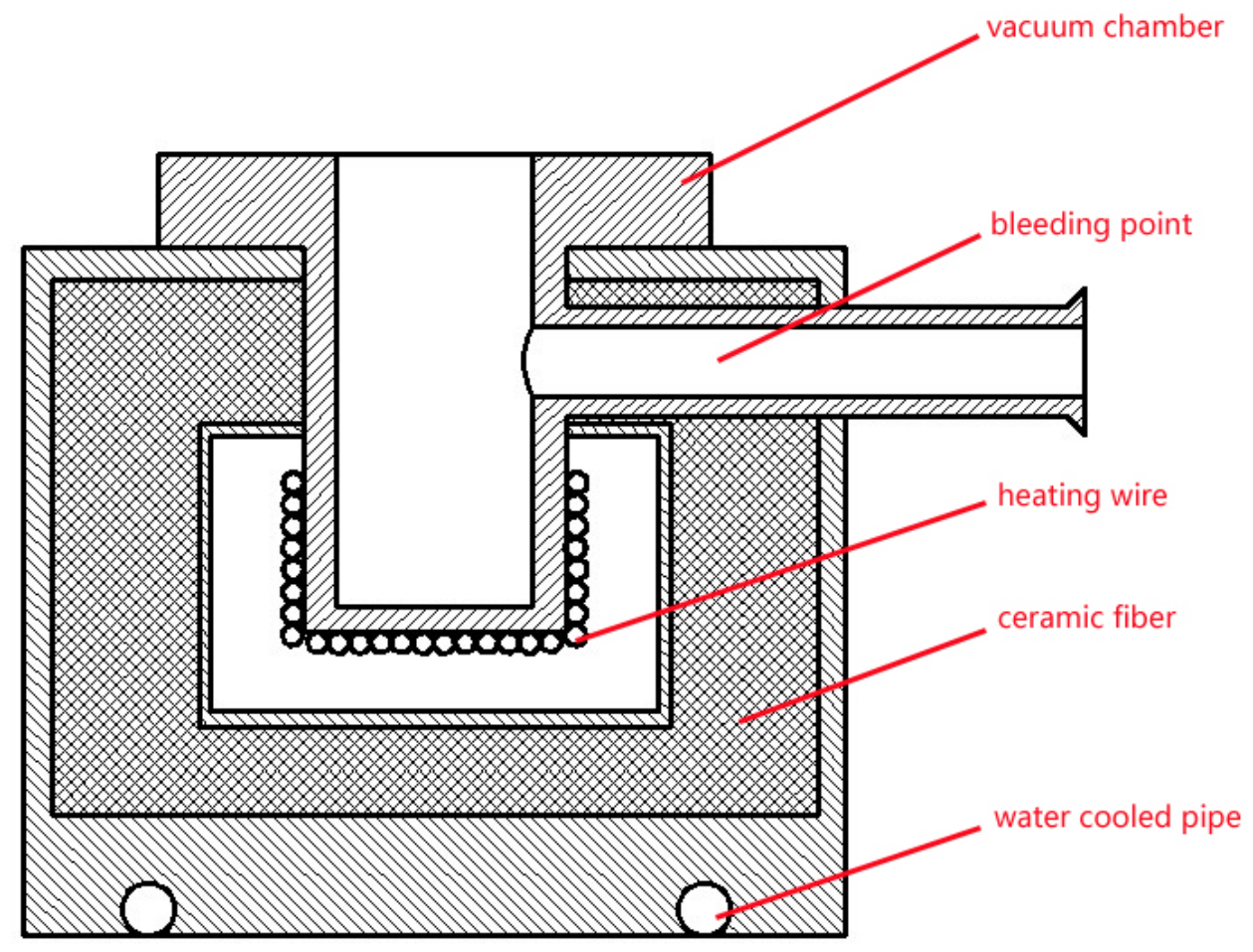

Fig. 3 The design of the heater

\section{Summary}

This paper detailed on the micro vacuum heating observation equipment. The vacuum system and heating system run extremely smoothly. It can realize the observation mission which in the vacuum and heating environment well. Vacuum degree requirements can be achieved. And the temperature accuracy can meet the requirements. The combination of vacuum chamber and heater provides the powerful guarantee for the microscope observation.

\section{Acknowledgements}

Project supported by "The Fundamental Research Funds of Shandong University" No.2014YQ006.

\section{References}

[1] ZHANG Zheng-bing. Design of Vacuum System of Large Plasma Processing Equipment, J. Electro-Mechanical Engineering. Vol.30(2014)No.4, p.24-29

[2] LIAN Fang. MOSFET Solid High-frequency Induction Heating Equipment and Its Application, J. Heat Treatment.Vol.29(2014)No.4,p.47-51

[3] Gao Ya-jie,XIE Yong-rong, ZHANG Yan-bin, et al. Development of ultra-high vacuum heating furnace, J. Vacuum. Vol.52(2015)No.5, p.58-60

[4] Zhengzhong Gao, Qingliang Zeng, Shiguang Li. Intelligent Vacuum-pumping Detection Device Base On STM32MCU, J. Applied Mechanics and Materials. Vol.190-191(2012),p.1002-1005 